\title{
Coupling radio propagation and weather forecast models to maximize Ka-band channel transmission rate for interplanetary missions
}

\author{
M. Biscarini ${ }^{(1)}$, F. S. Marzano ${ }^{(1)}$, M. Montopoli $^{(1)}$, L. Iess ${ }^{(2)}$, K. De Sanctis ${ }^{(3)}$, S. Di Fabio ${ }^{(3)}$, M. \\ Montagna $^{(4)}$, M. Mercolino ${ }^{(4)}$, and M. Lanucara ${ }^{(4)}$ \\ (1) DIET and CRAS, Sapienza University of Rome, Italy \\ (2) DIMA and CRAS, Sapienza University of Rome, Italy \\ (3) HIMET and CETEMPS, University of L'Aquila, Italy \\ (4) ESOC, European Space Agency, Germany
}

Deep space (DS) missions for interplanetary explorations are aimed at acquiring information about the solar system and its composition. To achieve this result a radio link is established between the space satellite and receiving stations on the Earth. Significant channel capacity must be guaranteed to such spacecraft-to-Earth link considering their large separation distance as well. Terrestrial atmospheric impairments on the space-to-Earth propagating signals are the major responsible for the signal degradation thus reducing the link's channel temporal availability. Considering the saturation and the limited bandwidth of the conventional systems used working at X-band (around $8.4 \mathrm{GHz}$ ), frequencies above Ku-band (12-18 GHz) are being used and currently explored for next future DS missions. For example, the ESA mission EUCLID, planned to be launched in 2020 to reach Sun-Earth Lagrange point L2, will use the K-band (at 25.5-27 GHz). The BepiColombo (BC) ESA mission to Mercury, planned to be launched in 2016, will use Ka-band (at 32-34 GHz) with some modules operating at X-band too. The W-band is also being investigated for space communications (Lucente et al., IEEE Systems J., 2008) as well as near-infrared band for DS links (Luini at al., $3^{\text {rd }}$ IWOW, 2014; Cesarone et al., ICSOS, 2011).

If compared with X-band channels, K-band and Ka-band can provide an appealing data rate and signal-to-noise ratio in free space due to the squared-frequency law increase of antenna directivity within the downlink budget (for the same physical antenna size). However, atmospheric path attenuation can be significant for higher frequencies since the major source of transmission outage is not only caused by convective rainfall, as it happens for lower frequencies too, but even non-precipitating clouds and moderate precipitation produced by stratiform rain events are detrimental. This means that accurate channel models are necessary for DS mission data link design at $\mathrm{K}$ and $\mathrm{Ka}$ band. A physical approach can offer advanced radiopropagation models to take into account the effects due to atmospheric gases, clouds and precipitation.

The objective of this work is to couple a weather forecast numerical model with a microphysicallyoriented radiopropagation model, providing a description of the atmospheric state and of its effects on a DS downlink. This work is developed in the framework of the RadioMeteorological Operations Planner (RMOP) program, aimed at performing a feasibility study for the BC mission (Biscarini et al., EuCAP 2014). The RMOP chain for the link budget computation is composed by three modules: weather forecast (WFM), radio propagation (RPM) and downlink budget (DBM). WFM is aimed at providing an atmospheric state vector. Among the available weather forecast models, for RMOP purposes we have used the Mesoscale Model 5. The output of the WFM is the input of the RPM for the computation of the atmospheric attenuation and sky-noise temperature at the receiving ground station antenna. RPM makes use of radiative transfer solver based on the Eddington approximations well as accurate scattering models. Time series of attenuation and sky-noise temperature coming from the RPM are converted into probability density functions and then ingested by the DBM to compute the received data volume (DV).

Using the $\mathrm{BC}$ mission as a reference test case for the Ka-band ground station at Cebreros (Spain), this work will show the advantages of using a coupled WFM-RPM approach with respect to climatological statistics in a link budget optimization procedure. The signal degradation due to atmospheric effects in DS links in terms of received DV will be also investigated not only at $\mathrm{Ka}$ band, but also at X, K and $\mathrm{W}$ for intercomparison. The quality of the DS downlink will be given in terms of received DV and the results at different frequencies compared showing the respective advantages and drawbacks. 